Journal of Computer Science 7 (4): 554-560, 2011

ISSN 1549-3636

(C) 2011 Science Publications

\title{
Analytical Modeling of IEEE 802.11e Enhanced Distributed Channel Access under a Non-Saturation Condition
}

\author{
${ }^{1}$ G. Prakash and ${ }^{2} \mathrm{P}$. Thangaraj \\ ${ }^{1}$ Department of Electronics and Communication Engineering, \\ K.S.R College of Technology, Nammakal, Tamil Nadu, India \\ ${ }^{2}$ Department of Computer Science and Engineering, BIT, \\ Sathyamanglam, Erode, Tamil Nadu, India
}

\begin{abstract}
Problem statement: The IEEE 802.11e EDCA protocol with different access Categories (ACs) supporting for Quality-of-Service (QoS). Due to internal or external packet collision, the Contention Window (CW) of the station increases the channel idle time under high Bit Error Rate (BER). Approach: In this study, we propose an analytical model for performance evaluation of IEEE 802.11e EDCA scheme under non-saturation condition and error prone channel. The new markov chain model have decrease the channel idle time in IEEE 802.11 EDCA and considerably increases the throughput for minimum number of station. Results: We develop an expression for the nonsaturation throughput as a function of the number of stations, packet sizes and BER. Conclusion: We validate the accuracy of our analysis with simulation expression. Using this model, the contention factors can be set appropriately to attain particular Quality-of-Service $(\mathrm{Q} o S)$ requirements.
\end{abstract}

Key words: Enhanced Distributed Channel Access (EDCA), markov model, Contention Window (CW), non-saturation, Quality-of-Servi (QoS), Bit Error Rate (BER)

\section{INTRODUCTION}

In recent years, the IEEE 802.11 Wireless Local Area Network (WLAN) has been widely deployed in Indoor wireless Communication and it includes detailed specification of both medium access control and physical layer. The most research area of these two layers is Medium Access Control (MAC) layer in the first sub layer of data link layer. To access the medium from physical layer using MAC layer will be coordinating the random access of the medium. In wireless LAN MAC protocols is to coordinate the access of wireless client station in shared medium using carrier sense multiple Access/Collision Avoidance (CSMA/CA) protocol for MAC layer (IEEE Std. 802.11, 1999).

The IEEE 802.11e MAC layer is to enhancing the quality of services in WLAN which provides a contention-based channel access function and a centrally controlled channel access function called the Hybrid Coordination Function (HCF). The HCF combines a distribution contention-based channel access mechanism, referred to as Enhanced Distributed Channel Access (EDCA) and a centralized pollingbased channel access mechanism referred to as HCF Controlled Channel Access (HCCA). In this article, we concentrate on EDCA protocol which defines multiple Access Categories (AC) services for each station. Different levels of services are provided to each AC includes Contention Window $(\mathrm{CW})$ sizes, Arbitration Interframe Space (AIFS) values and Transmit Opportunity (TXOP) limits for providing QoS to users. In this study we concentrate on the performance analysis of EDCA scheme, in the assumption of non ideal conditions and finite number of terminals.

According to the author's knowledge the first analytical model of DCF was proposed by Bianchi (2000). Bianchi proposed a Markov chain based model to evaluate saturation throughput, the mobile stations always have something to transmit (i.e., the saturation condition), assuming a finite number of stations and ideal channel conditions (no errors). While in (Shyang et al., 2005) the authors extended the Bianchi model in order to consider unsaturated traffic conditions by adding another state (idle) to represent the node with empty queue, not present in the original Bianchi's model, in which the station buffer is empty, after a successful completion of a packet transmission. In the modified model, the packet is discarded after $\mathrm{m}^{\text {th }}$ backoff stages, while in Bianchi's model, the station keeps iterating in the mth backoff stage until the packet

Corresponding Author: G. Prakash, Department of Electronics and Communication Engineering, K. S. Rangasamy College of Technology, Namakkal, Tamil Nadu, India 
gets successfully transmitted. There are some works on unsaturated traffic condition and post backoff stage for IEEE 802.11b (Malone et al., 2007; Dhanasekaran senthil kumar and Krishnan, 2010; Daneshgaran et al., 2008). However, these models are not based on simplifying assumptions that the IEEE 802.11 standard defines a Station Short Retry Count (SSRC), which is the maximum number of RTS transmission attempts that will be made before a frame is discarded Typically it is set to 5or 7 (IEEE Std. 802.11, 1999; Wu et al., 2002; Chatz imisios et al., 2004; Prakash and Thangaraj, 2010). In (Chatzimisios et al., 2004), the performance of IEEE 802.11 assumes that there is a finite transmission and presence of transmission error. In an adhoc network (Priakanth and Thangaraj, 2009), proposed an efficient packets scheduling and queuing algorithm by achieving better fairness in Medium Access Control (MAC) protocol. In (Murugan and Shanmugam, 2010), proposed a solution to identify the malicious node in both routing and MAC layer in the ahoc network using cumulative frequency based detection technique. Both articles referenced performances of MAC layer without markov model.

The majority of analytical work on the performance of 802.11e EDCA assumes that there is a finite retransmission limit (retry limit) and infinite retry limit under saturation and non-saturation condition using markov chain model (Huang et al., 2007; Wei et al., 2007; Kong et al., 2004; Xiao, 2005; Engelstad and Osterbo, 2005; Inan et al., 2009). The EDCF and provided performance studies via simulation. In (Huang et al., 2007), the performance of IEEE 802.11e has investigated in the error prone channel under saturation condition. Based on the Markov chain model for unsaturated condition (Wei et al., 2007), an analytical approach is proposed for throughput and delay of 802.11e EDCA. (Xiao, 2005; Kong et al., 2004) extended the bianchi markov model to analyze the CW and AIFS differentiation under saturation condition. In (Kong et al., 2004) Kong's model considered the difference of AIFS in back-off procedures, but neglected AIFS while the back-off procedure reminded one timeslot. In (Hui and Devtsilitios, 2005) the authors proposed a new unified performance model to saturation throughput and delay performance of the network. These models were not provided real network condition. A few studies proposed real network condition which is on non-saturation analysis but not considered channel error. Englestad (2005c) presented an analytical model for IEEE 802.11e with Virtual collision handler $(\mathrm{VCH})$ and post-backoff under non saturation condition. Inan et al. (2009) proposed 3dimensional DTMCs under unsaturated traffic which provide accurate treatment of AIFS, CW differentiation and EDCA TXOP. In (Pan and $\mathrm{Wu}, 2009)$ throughput of the EDCA is investigated under heterogeneous traffic. In this study, we propose a new markov chain model (proposed model) for analyzing EDCA protocol under non-saturation condition with error-prone channel. Due to internal (virtual) collision occurs within the station, the contention window size of the station is need not to be doubled for packet transmission. In case of transmission error due to fading or packet collision from other stations experiences external collision, the contention window size is doubled for every transmission. Based on this previous work of Xiao's model (2005), Senthilkumar and Krishnan (2010), we developed a Markov chain model for the EDCA analysis. The study is organized as follows. In this study, we present the new Markov chain model by extending the model proposed initially by Bianchi. Performance of the proposal scheme is analyzed after markov chain model explanation. We discuss numerical results and then finally we present conclusion of this study.

\section{MATERIALS AND METHODS}

Markov model for IEEE 802.11e EDCA: We consider a WLAN in Unsaturation (Nonsaturation) condition, ie., buffer of the transmitting station is empty, after a successful transmission. To analyze an EDCA protocol, we extend the previous works on following assumption: (1) real channel condition; (2) channel is prone to errors due to fading channel; (3) finite and fixed number $\mathrm{N}$ of contending stations. Traffics are categorizing into $\mathrm{N}$ different Access categories according to their QoS requirements. For simplicity, we summarize all parameters related with the nth AC have a subscript n. Fig.1 shows that the state of the nth AC by $\{n, i, k\}$. Here, $i,\left(i=0,1, \ldots ., m_{n}\right)$ represents the backoff stage, where $m_{n}$ denotes the retry limit. $\mathrm{k}\left(\mathrm{k}=0,1,2, \ldots, \mathrm{W}_{\mathrm{n}, \mathrm{i}}-1\right)$ represents the value of backoff counter, where $\mathrm{W}_{\mathrm{n}, \mathrm{i}}$ is the contention window size in the backoff stage $\mathrm{i}$ for the nth AC. The state (n, i, $\mathrm{k})$ represents the $\mathrm{i}^{\text {th }}$ backoff stage of nth $\mathrm{AC}$ with backoff counter value $\mathrm{k}$ and the $\mathrm{CW}$ size of backoff stage $i$ of nth AC is expressed as:

$\mathrm{W}_{\mathrm{n}, \mathrm{i}}=\left\{\begin{aligned} 2^{\mathrm{i}} \mathrm{W}_{\mathrm{n}, 0,} & 0 \leq \mathrm{i} \leq \mathrm{m}_{\mathrm{n}} \\ 2^{\mathrm{m}_{\mathrm{n}}} \mathrm{W}_{\mathrm{n}, 0}, & \mathrm{~m}_{\mathrm{n}}<\mathrm{i} \leq \mathrm{m}_{\mathrm{n}}^{\prime}\end{aligned}\right.$

where, $\mathrm{W}_{\mathrm{n}, \mathrm{o}}=\mathrm{CW}_{\mathrm{n}, \min }+1, \mathrm{~m}_{\mathrm{n}}$ is the backoff stage of which $\mathrm{CW}$ size is the maximum $\mathrm{CW}$ size and $\mathrm{m}_{\mathrm{n}}^{\prime}$ is the frame retry limit. Our main aim in this performance study is the effective modification of the EDCA MAC protocol in order to characterize the behavior of MAC in the event of channel errors. In the basic AC, the contention window is doubled after every unsuccessful transmission. 


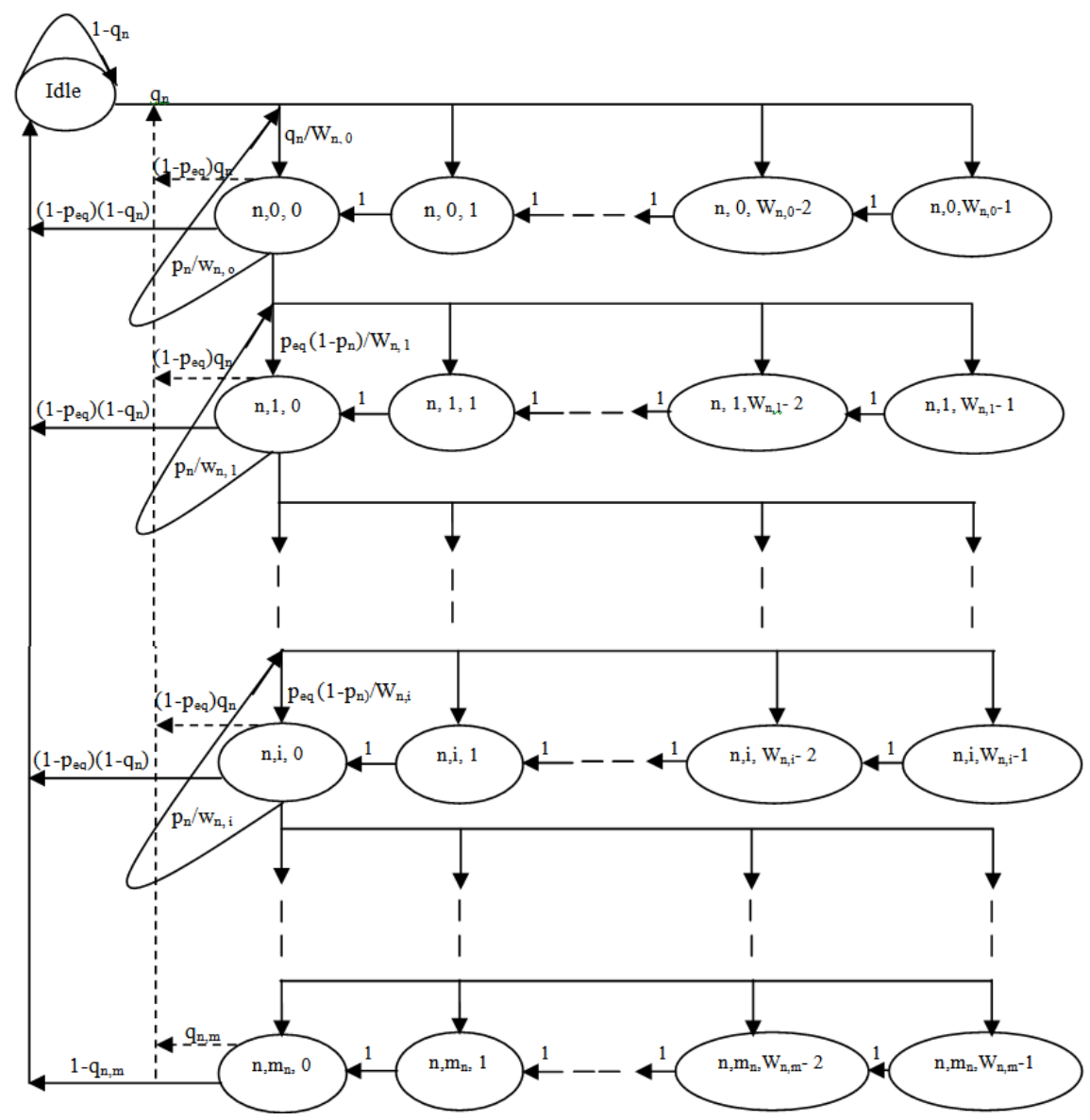

Fig. 1: Proposed Markov Chain Model for EDCA

Unsuccessful transmission happens in two cases: (i) internal collision or virtual collision of a packet with other packets with in a station, (ii) due to error in the channel and packet collision with other station. Let $b(t)$ be the stochastic process that represents the backoff timer for a specific station and $\mathrm{s}(\mathrm{t})$ be the stochastic process representing the backoff stage $[0, \ldots ., \mathrm{m}]$ for a given station at time $t$; where $m$ is the packet retry limit. Our analysis assumes that the network consists of $n$ Contending stations and that each station has not always a packet available for transmission. The key assumption of our model is that the collision-error probability $\left(\mathrm{p}_{\text {col }}\right)$ and transmission error $\left(\mathrm{p}_{\mathrm{e}}\right)$ of a transmitted packet is constant and independent of the number of collisions or transmission errors of this packet has suffered in the past. We assume that collisions (internal collision) and transmission error (external collision) events are statistically independent. The three dimensional discrete time Markov chain is depicted in Fig. 1. In this Markov chain, the only nonnull one step transition probabilities are as follows:
$\mathrm{P}(\mathrm{n}, \mathrm{i}, \mathrm{k} / \mathrm{n}, \mathrm{i}, \mathrm{k}+\mathrm{l})=1$,

$\mathrm{k} \in\left[0, \mathrm{~W}_{\mathrm{n}, \mathrm{i}}-1\right), \mathrm{i} \in\left[0, \mathrm{~m}_{\mathrm{n}}\right]$

$\mathrm{P}(\mathrm{n}, 0, \mathrm{k} / \mathrm{n}, \mathrm{i}, 0)=\frac{\mathrm{q}_{\mathrm{n}}\left(1-\mathrm{p}_{\mathrm{eq}}\right)}{\mathrm{W}_{\mathrm{n}, 0}}$,

$\mathrm{k} \in\left[0, \mathrm{~W}_{\mathrm{n}, 0}-1\right], \quad \mathrm{i} \in\left[0, \mathrm{~m}_{\mathrm{n}}\right]$

$\mathrm{P}(\mathrm{n}, \mathrm{i}, \mathrm{k} / \mathrm{n}, \mathrm{i}, 0)=\frac{\mathrm{p}_{\mathrm{n}}}{\mathrm{W}_{\mathrm{n}, \mathrm{i}}}$,

$\mathrm{k} \in\left[0, \mathrm{~W}_{\mathrm{n}, \mathrm{i}}-1\right], \quad \mathrm{i} \in\left[0, \mathrm{~m}_{\mathrm{n}}-1\right]$

$\mathrm{P}(\mathrm{n}, \mathrm{i}, \mathrm{k} / \mathrm{n}, \mathrm{i}-1,0)=\frac{\mathrm{p}_{\mathrm{eq}}\left(1-\mathrm{p}_{\mathrm{n}}\right)}{\mathrm{W}_{\mathrm{n}, \mathrm{i}}}$,

$\mathrm{k} \in\left[0, \mathrm{~W}_{\mathrm{n}, \mathrm{i}}-1\right], \mathrm{i} \in\left[1, \mathrm{~m}_{\mathrm{n}}\right]$

If there is no packet in a buffer after a successful transmission, the station is waiting in the idle state until a new packet arrives:

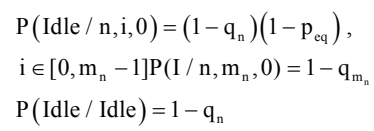


The first Eq. in (2), beginning of each slot time, backoff time is decremented. The second equation in (2) states that, the initialization of backoff window after successful transmission for a new packet. The new value of the backoff timer is uniformly chosen in the interval $\left(0, \mathrm{~W}_{\mathrm{n}, 0}-2\right)$. The third equation represent that the probability of unsuccessful transmission due to internal collision $\left(\mathrm{p}_{\mathrm{n}}\right)$, but maintenance the same backoff window. The fourth equation accounts that, probability of unsuccessful transmission due to external collision or channel error $\left(p_{\text {eq }}\right)$. In Eq. (2) $p_{\text {eq }}$ represents the external collsion and channel errors, $p_{n}$ represents that the probability due to internal collision and q represents the probability of atleast one packet awaiting transmission at the start of a counter decrement. The probability of successful transmission is $\left(1-q_{n}\right)\left(1-p_{e q}\right)$. Let $b_{n, i, k}=\lim$ $t \rightarrow \infty \mathrm{P}[\mathrm{s}(\mathrm{n}, \mathrm{t})=\mathrm{i}, \mathrm{b}(\mathrm{n}, \mathrm{t})=\mathrm{k}]$ be the stationary distribution of the Markov chain. In steady state, we can derive the following relations through chain regularities.

Analysis and Throughput Computation:

$b_{n, i, 0}=p_{e q}\left(1-p_{n}\right) b_{n, i-1,0}+p_{n} b_{n, i, 0}, i \in\left(0, m_{n}\right)$

$b_{n, i, 0}=P^{i} \cdot b_{n, 0,0} \quad i \in\left(0, m_{n}\right)$

Where $P=\frac{p_{e q}\left(1-p_{n}\right)}{p_{n}}$,

$\mathrm{p}_{\mathrm{eq}}$ (probabilit y of failed transmission $)=\mathrm{p}_{\mathrm{e}}+\mathrm{p}_{\mathrm{col}}-\mathrm{p}_{\mathrm{e}} \mathrm{p}_{\text {col }}$ :

$b_{\text {idle }}=\frac{\left(1-p_{e q}\right)\left(1-q_{n}\right)}{q_{n}} \sum_{i=0}^{m_{n}} b_{n, i, 0}+\left(1-q_{n}\right) p_{e q}^{m_{n}+1} \cdot b_{n, 0,0}$

All the steady state probabilities of the markov chain are expressed as function of $b_{n, 0,0}$ and it can be obtained from this normalization equation:

$$
1=\sum_{\mathrm{i}=0}^{\mathrm{m}_{\mathrm{n}}} \sum_{\mathrm{k}=0}^{\mathrm{w}_{\mathrm{i}, \mathrm{j}}-1} \mathrm{~b}_{\mathrm{n}, \mathrm{i}, \mathrm{k}}+\mathrm{b}_{\mathrm{idle}}
$$

We know that:

$$
\mathrm{b}_{\mathrm{n}, \mathrm{i}, \mathrm{o}}=\mathrm{p}_{\mathrm{eq}}^{\mathrm{i}} \cdot \mathrm{b}_{\mathrm{n}, 0,0}
$$

$$
\begin{aligned}
\mathrm{b}_{\mathrm{n}, 0,0}= & \frac{2(1-2 \mathrm{P})(1-\mathrm{P}) \mathrm{q}_{\mathrm{n}}}{\mathrm{W}_{\mathrm{n}, 0}\left(1-(2 \mathrm{P})^{\mathrm{m}_{\mathrm{a}}+1}\right)(1-\mathrm{P}) \mathrm{q}_{\mathrm{n}}+} \\
& (1-2 \mathrm{P})\left(1-\mathrm{P}^{\mathrm{m}_{\mathrm{a}}+1}\right) \mathrm{q}_{\mathrm{n}}+ \\
& \mathrm{W}_{\mathrm{n}, 0} \cdot 2^{\mathrm{m}^{\prime}} \cdot \mathrm{P}^{\mathrm{m}_{\mathrm{a}}+1}(1-2 \mathrm{P}) \mathrm{q}_{\mathrm{n}}\left(1-\mathrm{P}^{\mathrm{m}_{\mathrm{n}}-\mathrm{m}_{\mathrm{n}}^{\prime}}\right)+ \\
& \left(1-\mathrm{q}_{\mathrm{n}}\right)(1-2 \mathrm{P})(1-\mathrm{P})\left(1-\mathrm{P}^{\mathrm{m}_{\mathrm{a}}-1}\right) \mathrm{m}_{\mathrm{n}}>\mathrm{m}_{\mathrm{n}}^{\prime}
\end{aligned}
$$

$$
\begin{aligned}
\mathrm{b}_{\mathrm{n}, 0,0}= & \frac{2(1-2 \mathrm{P})(1-\mathrm{P}) \mathrm{q}_{\mathrm{n}}}{1-(2 \mathrm{P})^{\mathrm{m}_{\mathrm{n}}+1} \cdot \mathrm{W}_{\mathrm{n}, 0}(1-\mathrm{P}) \mathrm{q}_{\mathrm{n}}} \\
& +\left(1-\mathrm{P}^{\mathrm{m}_{\mathrm{n}}+1}\right)(1-2 \mathrm{P}) \mathrm{q}_{\mathrm{n}} \\
& +\left(1-\mathrm{P}^{\mathrm{m}_{\mathrm{n}}+1}\right)\left(1-\mathrm{q}_{\mathrm{n}}\right) \\
& +\left(1-\mathrm{q}_{\mathrm{n}}\right) \cdot \mathrm{P}^{\mathrm{m}_{\mathrm{n}}+1} \cdot \mathrm{q}_{\mathrm{n}}(1-2 \mathrm{P})(1-\mathrm{P}) \mathrm{m}_{\mathrm{n}} \leq \mathrm{m}_{\mathrm{n}}^{\prime}
\end{aligned}
$$

Let $\tau_{\mathrm{n}}$ denote the transmission probability can be written as:

$$
\tau_{n}=\sum_{i=0}^{m_{n}} b_{n, i, 0}=\frac{1-P^{m_{n}+1}}{1-P} \cdot b_{n, 0,0} \quad 1 \leq n \leq N_{t}
$$

A transmitted frame collides when two or more stations transmit during slot time, so the probability $p_{n}$ that transmitted frame collides and channel busy probability $\mathrm{p}_{\mathrm{b}}$ in a slot can be expressed as:

$$
\begin{aligned}
& \mathrm{p}_{\mathrm{n}}=1-\left(1-\tau_{\mathrm{n}}\right)^{\mathrm{N}} \prod_{\mathrm{j}=0}^{\mathrm{N}_{\mathrm{t}}-1}\left(1-\tau_{\mathrm{j}}\right)^{\mathrm{n}_{\mathrm{j}}} \\
& \mathrm{p}_{\mathrm{b}}=1-\prod_{\mathrm{i}=0}^{\mathrm{N}_{\mathrm{t}}-1}\left(1-\tau_{\mathrm{i}}\right)^{\mathrm{N}}
\end{aligned}
$$

The values of $P$ and $\tau$ are still unknown in above Eq. 8-9 which can be solved using numerical techniques and represents as nonlinear equations. The probability that no station is transmitting in the slot time is expressed as:

$$
\mathrm{P}_{\mathrm{idle}}=\prod_{\mathrm{i}=0}^{\mathrm{N}_{\mathrm{t}}-1}\left(1-\tau_{\mathrm{i}}\right)^{\mathrm{N}}=1-\mathrm{p}_{\mathrm{b}}
$$

Non-Saturation Throughput: Let $\mathrm{P}_{\mathrm{n}, \mathrm{s}}$ be the probability that a successful transmission occurs in a slot time of the nth $\mathrm{AC}$ and $\mathrm{P}_{\mathrm{s}}$ denote the probability that successful transmission occurs in a slot time. We have:

$$
\begin{aligned}
& P_{n, s}=\tau_{n}\left(1-\tau_{n}\right)^{n_{n}-1} \prod_{j=0}^{N_{t}-1}\left(1-\tau_{j}\right)^{n_{j}} \\
& P_{s}=\sum_{n=0}^{N_{t}-1} P_{n, s}=\sum_{n=0}^{N_{t}-1} \frac{n_{n} \tau_{n}}{1-\tau_{n}}\left(1-p_{b}\right)
\end{aligned}
$$

The normalized throughput of $n$th $\mathrm{AC}, \mathrm{S}_{\mathrm{n}}$ is: 
$\mathrm{S}_{\mathrm{n}}=\frac{\text { [payload inf ormation successfully transmitted in slot time] }}{\text { [Average length of a time slot] }}$

$S_{n}=\frac{P_{n, s}\left(1-p_{e}\right) \cdot \text { PACKET }}{\left(1-p_{b}\right) \sigma+P_{s}\left(1-p_{e}\right) T_{s}+\left(p_{b}-P_{s}\right) T_{c}+P_{s} \cdot p_{e} \cdot T_{e}}$

PACKET includes PHY header, MAC header and data payload length of the nth AC. $T_{n, s}=T_{s}$ and $T_{n, c}=T_{c}$ are the successful and unsuccessful transmission time. $T_{e}$ is the unsuccessful transmission time due to transmission errors. The value of $T_{s}, T_{c}$ and $T_{e}$ can be expressed as:

$\mathrm{T}_{\mathrm{n}, \mathrm{s}}=\mathrm{PACKET}+\mathrm{ACK}+2 \delta+\operatorname{SIFS}+\operatorname{AIFS}[\mathrm{n}]$

$\mathrm{T}_{\mathrm{n}, \mathrm{c}}=\mathrm{RTS}+\operatorname{AIFS}[\mathrm{n}]+\delta$

$\mathrm{T}_{\mathrm{e}}=\mathrm{PACKET}+2 \delta+\mathrm{SIFS}+\mathrm{AIFS}[\mathrm{n}]+\mathrm{ACK}$ Timeout

ACK is the time to transmit the Acknowledgement (ACK) packet, AIFS[n] is the time of AIFS of priority $n$ class, RTS is the time to transmit the packet RTS, CTS is the time to transmit the packet CTS and $\delta$ is the propagation delay. In our analysis, we consider Poisson process for packet arrival. $\mathrm{q}_{\mathrm{n}}$ is the probability that indicates if there is at least one packet to be transmitted in queue during the time slot under the condition that the queue is empty at the beginning of the slot. For unsaturation condition, the probability $q$ can be expressed as:

$\mathrm{q}_{\mathrm{n}}=1-\mathrm{e}^{-\lambda_{\mathrm{n}} \mathrm{E}[\mathrm{slot}]}$

Where $\quad E[$ slot $]=\left(1-p_{b}\right) \cdot \sigma+P_{s}\left(1-p_{e}\right) \cdot T_{s}+\left(p_{b}-P_{s}\right) T_{c}+P_{s} p_{e} T_{e}$ and $\lambda_{n}$ is the Poisson arrival rate of traffic to $\mathrm{AC}[\mathrm{n}]$.

\section{RESULTS AND DISCUSSION}

In this study, we present numerical results that show the impact of transmission errors on the system capacity. The network parameters used in these results are shown in Table 1 and we assume every station is able to listen each other and there are no hidden terminals in the systems. The Figure validates our mathematical analysis since an almost exact match is observed between analytical (symbols) and simulation results (lines) from our IEEE 802.11e simulator developed with the NS-2 simulation package. Figure 2 compares number of stations for each AC per station against throughput. In this graph, the highest priority of $\mathrm{AC}$ shows that higher throughput for fixed packed length.

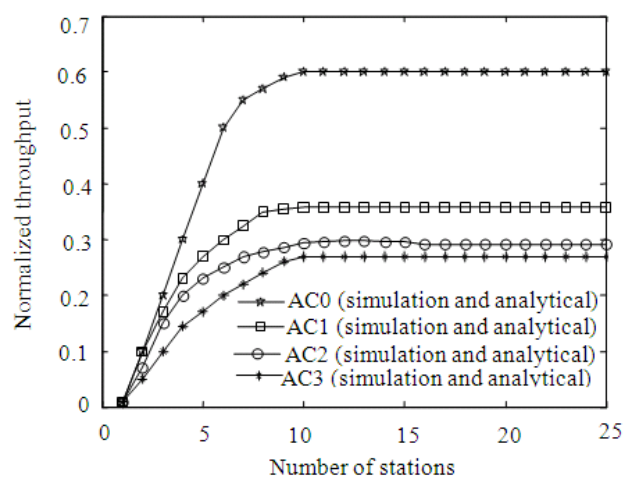

Fig. 2: Numerical and simulation results for normalized throughput

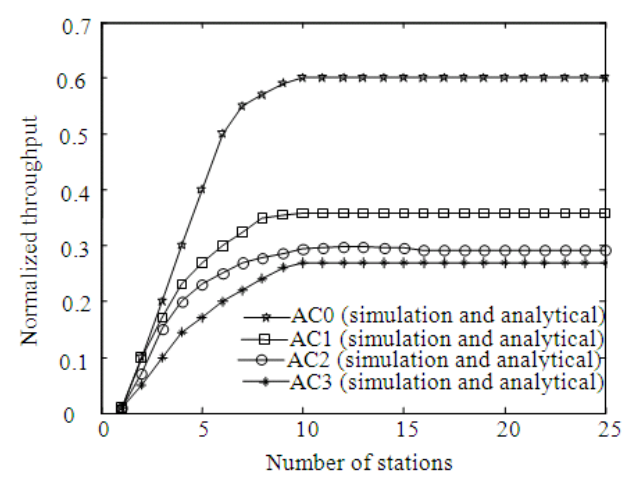

Fig. 3: Throughput versus number of stations for $\mathrm{p}_{\mathrm{e}}=0.0001$

Table 1: Calculation and Simulation Parameter

\begin{tabular}{ll}
\hline PHY header & 192 bits \\
MAC header & 272 bits \\
RTS frame & PHY header +160 bits \\
CTS frame & PHY header +112 bits \\
ACK timeout & DIFS + ACK \\
Date frame & $2 \mathrm{Mbps}$ \\
Slot time & $20 \mu \mathrm{s}$ \\
SIFS & $10 \mu \mathrm{s}$ \\
DIFS & SIFS +2. aSlotTime \\
AIFSN & $\{2,2,3,7\}$ \\
Propagation delay & $1 \mu \mathrm{s}$ \\
CW $_{\min }$ & $\{7,15,31,31\}$ \\
$\mathrm{CW}_{\max }$ & $\{15,31,1023,1023\}$ \\
\hline
\end{tabular}

Figure 3 shows the throughput versus number of station with probability of error. Here, the channel bit rate is increases and the throughput slightly decreases with increased networks. Figure 4 compares the simulation and analytical results of modified model by plotting the throughput against different packet arrival rate for transmission error channel. In this graph, the low traffic of all AC shows that the modified model 


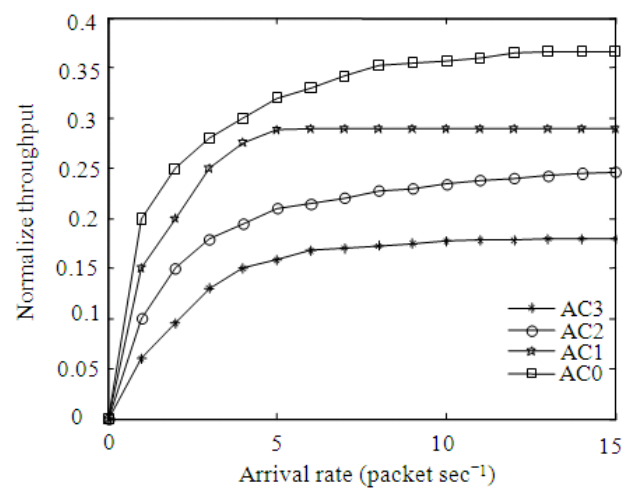

Fig. 4: Throughput versus packet arrival rate

significantly increases the throughput. From above results, the number of higher priority ACs is $\mathrm{AC} 0$ gives an acceptable throughput performance for all situation.

\section{CONCLUSION}

In this study, we have presented an analytical model that calculates throughput of the IEEE 802.11e EDCA protocol. We have presented an accurate analytical model to evaluate the performance of EDCA under nonsaturation conditions. Simulation and analysis results show that our analytical model can accurately predict the throughput performance of IEEE 802.11e EDCA under nonsaturation conditions in presence of channel errors. Based on this model we derive a novel and generalized expression for the station's transmission probability, which is more realistic, such as traffic load conditions (different ACs) and non ideal channel conditions. Our study shows that an optimally chosen number of contending stations can significantly improves the network throughput.

\section{REFERENCES}

Bianchi, G., 2000. Performance analysis of the IEEE 802.11 distributed coordination function. IEEE J. Select. Areas Commun., 18: 535-547. DOI: 10.1109/49.840210

Chatzimisios, P., A.C. Boucouvalas and V. Vitsas, 2004. Performance analysis of IEEE 802.11 DCF in presence of transmission Errors. Proceedings of the IEEE International Conference on Communications, June 20-24, Bournemouth University, UK., pp: 3854-3858. DOI: 10.1109/ICC.2004.1313274
Daneshgaran, F., M. Laddomada, F. Mesiti and M. Mondin, 2008. Unsaturated throughput analysis of IEEE 802.11 in presence of non ideal transmission channel and capture effects. IEEE Trans. Wireless Commun., 7 : 1276-1286. DOI: 10.1109/TWC.2008.060859

Engelstad, P.E. and O.N. Osterbo, 2005. Non-saturation and saturation analysis of IEEE 802.11e EDCA with starvation prediction. Proceedings of the 8th ACM International Symposium on Modeling, Analysis and Simulation of Wireless and Mobile Systems, (MSWIM'05), ACM New York, NY, USA., $\quad$ pp: 224-233. DOI: 10.1145/1089444.1089485

Huang, A., L. Shen, J. Li and T. Tang, 2007. Modeling and performance evaluation of IEEE 802.11e EDCA in Error-prone channel conditions. Proceeding of the IEEE Conference on Global Telecommunication, Nov. 26-30, IEEE Xplore, Washington, pp: 1344-1348. DOI: $10.1109 /$ GLOCOM.2007.258

Hui, J. and M. Devtsilitios, 2005. A unified model for the performance analysis of IEEE 802.11e EDCA. IEEE Trans. Commun., 53: 1498-1510. DOI: 10.1109/TCOMM.2005.855013

IEEE Std. 802.11, 1999. Wireless LAN Medium Access Control (MAC) and Physical Layer (PHY) Specification: High-speed Physical Layer in the 5 GHZ Band. The Institute of Electrical and Electronics Engineers, Inc., USA. http://pdos.csail.mit.edu/decouto/papers/802.11a.pdf

Inan, I., F. Keceli and E. Ayanoglu, 2009. Analysis of the 802.11e enhanced distributed channel access function. IEEE Trans. Commun., 57: 1753-1764. DOI: 10.1109/TCOMM.2009.06.0701132

Kong, Z., D.H.K. Tsang, B. Bensaou and D. Guo, 2004. Performance analysis of IEEE 802.11e contentionbased channel access. IEEE J. Select. Areas Commun, 22: 2095-2106. DOI: 10.1109/JSAC.2004.836019

Malone, D., K. Duffy and D.J. Leith, 2007. Modeling the 802.11 distributed coordination function in nonsaturated heterogeneous conditions. IEEE/ACM Trans. Network., 15: 159-172. DOI: 10.1109/TNET.2006.890136

Murugan, R. and A. Shanmugam, 2010. A combined solution for routing and medium access control layer attacks in mobile ad hoc networks. J. Comput. Sci., 6: 1416-1423. DOI: 10.3844/jcssp.2010.1416.1423

Pan, S.W. and J.S. Wu, 2009. Throughput analysis of IEEE 802.11e EDCA under heterogeneous traffic. Comput. Communun., 32: 935-942. DOI: 10.1016/j.comcom.2008.12.021 
Prakash, G. and .P. Thangaraj, 2010. Throughput analysis of IEEE $802.11 \mathrm{~b}$ WLAN under a NonSaturated condition. Proceedings of the International Conference on Recent Trends in Information, Telecommunication and Computing, Mar. 12-13, Kochi, Kerala, India, pp: 65-69. http://www.computer.org/portal/web/csdl/doi/10.11 09/ITC.2010.34

Priakanth, P. and P. Thangaraj, 2009. A channel adaptive energy efficient and fair scheduling media access control protocol for mobile Adhoc networks. J. Comput. Sci., 5: 57-63. DOI: 10.3844/jcssp.2009.57.63

Senthilkumar, D. and A. Krishnan, 2010. Nonsaturation throughput enhancement of IEEE $802.11 \mathrm{~b}$ distributed coordination function for heterogeneous traffic under noisy environment. Int. J. Automation Comput., 7: 95-104. DOI: 10.1007/s11633-0100095-6

Shyang, L.Y, A. Dadej and A. Jayasuriya, 2005. Performance analysis of IEEE 802.11 DCF under limited load. Proceedings of the Asia-Pacific Conference on Communications, Oct. 5-5, IEEE Xplore, Perth, WA., pp: 759-763. DOI: 10.1109/APCC.2005.1554164
Wei, Z., S. Jun, L. Jing and Z. Hai-bin, 2007. Performance analysis of IEEE 802.11e EDCA in wireless LANs. J. Zhejiang Univ.-Sci. A., 8: 18-23. DOI: 10.1631/jzus.2007.A0018

Wu, H., Y. Peng, K. Long, S. Cheng and J. Ma, 2002. Performance of reliable transport protocol over IEEE 802.11 Wireless LAN: Analysis and Enhancement. Proceedings of the 21st Annual Joint Conference of the IEEE Computer and Communications Societies, (INFOCOM'02), Beijing University of Posts and Telecommunication, Beijing, pp: 599-607. DOI: 10.1109/INFCOM.2002.1019305

Xiao, Y., 2005. Performance analysis of priority schemes for IEEE 802.11 and IEEE 802.11e wireless LANs. IEEE Trans. Wirel. Commun., 4: 1506-1515. DOI: 10.1109/TWC.2005.850328 